\title{
Common Coupled Fixed-Point Theorems in Generalized Fuzzy Metric Spaces
}

\author{
K. P. R. Rao, ${ }^{1}$ I. Altun, ${ }^{2}$ and S. Hima Bindu ${ }^{3}$ \\ ${ }^{1}$ Department of Mathematics, Acharya Nagarjuna University, Dr. M.R. Appa Row Campus, Nuzvid 521 201, India \\ ${ }^{2}$ Department of Mathematics, Faculty of Science and Arts, Kirikkale University, 71450 Yahsihan, Turkey \\ ${ }^{3}$ Department of Mathematics, CH. S.D. St. Theresa's Junior College for Women, Eluru 534 001, India
}

Correspondence should be addressed to K. P. R. Rao, kprrao2004@yahoo.com

Received 9 August 2011; Accepted 2 November 2011

Academic Editor: E. E. Kerre

Copyright ( $) 2011$ K. P. R. Rao et al. This is an open access article distributed under the Creative Commons Attribution License, which permits unrestricted use, distribution, and reproduction in any medium, provided the original work is properly cited.

We prove two unique common coupled fixed-point theorems for self maps in symmetric $G$-fuzzy metric spaces.

\section{Introduction and Preliminaries}

Mustafa and Sims [1-3] and Naidu et al. [4] demonstrated that most of the claims concerning the fundamental topological structure of $D$-metric introduced by Dhage [5-8] and hence all theorems are incorrect. Alternatively, Mustafa and Sims $[1,2]$ introduced a $G$-metric space and obtained some fixed-point theorems in it. Some interesting references in $G$-metric spaces are $[3,9-15]$. In this paper, we prove two unique common coupled fixed-point theorems for Jungck type and for three mappings in symmetric $G$-fuzzy metric spaces.

Before giving our main results, we recall some of the basic concepts and results in $G$-metric spaces and $G$-fuzzy metric spaces.

Definition 1 (see [2]). Let $X$ be a nonempty set and let $G$ : $X \times X \times X \rightarrow[0, \infty)$ be a function satisfying the following properties:

$\left(G_{1}\right) G(x, y, z)=0$ if $x=y=z$,

$\left(G_{2}\right) 0<G(x, x, y)$ for all $x, y \in X$ with $x \neq y$,

$\left(G_{3}\right) G(x, x, y) \leq G(x, y, z)$ for all $x, y, z \in X$ with $y \neq z$,

$\left(G_{4}\right) G(x, y, z)=G(x, z, y)=G(y, z, x)=\cdots$, symmetry in all three variables,

$\left(G_{5}\right) G(x, y, z) \leq G(x, a, a)+G(a, y, z)$ for all $x, y, z, a \in X$.

Then, the function $G$ is called a generalized metric or a $G$-metric on $X$ and the pair $(X, G)$ is called a $G$-metric space.
Definition 2 (see [2]). The $G$-metric space $(X, G)$ is called symmetric if $G(x, x, y)=G(x, y, y)$ for all $x, y \in X$.

Definition 3 (see [2]). Let $(X, G)$ be a $G$-metric space and let $\left\{x_{n}\right\}$ be a sequence in $X$. A point $x \in X$ is said to be limit of $\left\{x_{n}\right\}$ if and only if $\lim _{n, m \rightarrow \infty} G\left(x, x_{n}, x_{m}\right)=0$. In this case, the sequence $\left\{x_{n}\right\}$ is said to be $G$-convergent to $x$.

Definition 4 (see [2]). Let $(X, G)$ be a $G$-metric space and let $\left\{x_{n}\right\}$ be a sequence in $X .\left\{x_{n}\right\}$ is called G-Cauchy if and only if $\lim _{l, n, m \rightarrow \infty} G\left(x_{l}, x_{n}, x_{m}\right)=0 .(X, G)$ is called $G$-complete if every $G$-Cauchy sequence in $(X, G)$ is $G$-convergent in $(X, G)$.

Proposition 5 (see [2]). In a $G$-metric space $(X, G)$, the following are equivalent.

(i) The sequence $\left\{x_{n}\right\}$ is G-Cauchy.

(ii) For every $\epsilon>0$, there exists $N \in \mathbf{N}$ such that $G\left(x_{n}, x_{m}, x_{m}\right)<\epsilon$, for all $n, m \geq N$.

Proposition 6 (see $[2])$. Let $(X, G)$ be a G-metric space. Then, the function $G(x, y, z)$ is jointly continuous in all three of its variables.

Proposition 7 (see [2]). Let $(X, G)$ be a G-metric space. Then, for any $x, y, z, a \in X$, it follows that

(i) if $G(x, y, z)=0$, then $x=y=z$,

(ii) $G(x, y, z) \leq G(x, x, y)+G(x, x, z)$, 
(iii) $G(x, y, y) \leq 2 G(x, x, y)$,

(iv) $G(x, y, z) \leq G(x, a, z)+G(a, y, z)$,

(v) $G(x, y, z) \leq(2 / 3)[G(x, a, a)+G(y, a, a)+G(z, a, a)]$.

Proposition 8 (see [2]). Let $(X, G)$ be a $G$-metric space. Then, for a sequence $\left\{x_{n}\right\} \subseteq X$ and a point $x \in X$, the following are equivalent:

(i) $\left\{x_{n}\right\}$ is G-convergent to $x$,

(ii) $G\left(x_{n}, x_{n}, x\right) \rightarrow 0$ as $n \rightarrow \infty$,

(iii) $G\left(x_{n}, x, x\right) \rightarrow 0$ as $n \rightarrow \infty$,

(iv) $G\left(x_{m}, x_{n}, x\right) \rightarrow 0$ as $m, n \rightarrow \infty$.

Recently, Sun and Yang [16] introduced the concept of $G$-fuzzy metric spaces and proved two common fixed-point theorems for four mappings.

Definition 9 (see [16]). A 3-tuple $(X, G, *)$ is called a $G$ fuzzy metric space if $X$ is an arbitrary nonempty set, $*$ is a continuous $t$-norm, and $G$ is a fuzzy set on $X^{3} \times(0, \infty)$ satisfying the following conditions for each $t, s>0$ :

(i) $G(x, x, y, t)>0$ for all $x, y \in X$ with $x \neq y$,

(ii) $G(x, x, y, t) \geq G(x, y, z, t)$ for all $x, y, z \in X$ with $y \neq z$,

(iii) $G(x, y, z, t)=1$ if and only if $x=y=z$,

(iv) $G(x, y, z, t)=G(p(x, y, z), t)$, where $p$ is a permutation function,

(v) $G(x, y, z, t+s) \geq G(a, y, z, t) * G(x, a, a, s)$ for all $x, y, z, a \in X$,

(vi) $G(x, y, z, \cdot):(0, \infty) \rightarrow[0,1]$ is continuous.

Definition 10 (see [16]). A $G$-fuzzy metric space $(X, G, *)$ is said to be symmetric if $G(x, x, y, t)=G(x, y, y, t)$ for all $x, y \in X$ and for each $t>0$.

Example 11. Let $X$ be a nonempty set and let $G$ be a $G$-metric on $X$. Denote $a * b=a b$ for all $a, b \in[0,1]$. For each $t>0$, $G(x, y, z, t)=t /(t+G(x, y, z))$ is a $G$-fuzzy metric on $X$.

Let $(X, G, *)$ be a $G$-fuzzy metric space. For $t>0,0<$ $r<1$, and $x \in X$, the set $B_{G}(x, r, t)=\{y \in X: G(x, y, y, t)>$ $1-r\}$ is called an open ball with center $x$ and radius $r$.

A subset $A$ of $X$ is called an open set if for each $x \in$ $X$, there exist $t>0$ and $0<r<1$ such that $B_{G}(x, r, t) \subseteq A$.

A sequence $\left\{x_{n}\right\}$ in $G$-fuzzy metric space $X$ is said to be $G$-convergent to $x \in X$ if $G\left(x_{n}, x_{n}, x, t\right) \rightarrow 1$ as $n \rightarrow$ $\infty$ for each $t>0$. It is called a $G$-Cauchy sequence if $G\left(x_{n}, x_{n}, x_{m}, t\right) \rightarrow 1$ as $n, m \rightarrow \infty$ for each $t>0 . X$ is called $G$-complete if every $G$-Cauchy sequence in $X$ is $G$-convergent in $X$.

Lemma 12 (see [16]). Let $(X, G, *)$ be a $G$-fuzzy metric space. Then, $G(x, y, z, t)$ is nondecreasing with respect to $t$ for all $x, y, z \in X$.

Lemma 13 (see [16]). Let $(X, G, *)$ be a G-fuzzy metric space. Then, $G$ is a continuous function on $X^{3} \times(0, \infty)$.
Now onwards, we assume the following condition:

$$
\lim _{t \rightarrow \infty} G(x, y, z, t)=1 \quad \forall x, y, z \in X .
$$

Using $(\mathrm{P})$, one can prove the following lemma.

Lemma 14. Let $(X, G, *)$ be a $G$-fuzzy metric space. If there exists $k \in(0,1)$ such that

$$
\begin{aligned}
\min & \{G(x, y, z, k t), G(u, v, w, k t)\} \\
& \geq \min \{G(x, y, z, t), G(u, v, w, t)\}
\end{aligned}
$$

for all $x, y, z, u, v, w \in X$ and $t>0$, then $x=y=z$ and $u=v=w$.

Definition 15 (see [17]). Let $X$ be a nonempty set. An element $(x, y) \in X \times X$ is called a coupled fixed point of the mapping $F: X \times X \rightarrow X$ if $x=F(x, y)$ and $y=F(y, x)$.

Definition 16 (see [18]). Let $X$ be a nonempty set. An element $(x, y) \in X \times X$ is called

(i) a coupled coincidence point of $F: X \times X \rightarrow X$ and $g: X \rightarrow X$ if $g x=F(x, y)$ and $g y=F(y, x)$,

(ii) a common coupled fixed point of $F: X \times X \rightarrow X$ and $g: X \rightarrow X$ if $x=g x=F(x, y)$ and $y=g y=F(y, x)$.

Definition 17 (see [18]). Let $X$ be a nonempty set. The mappings $F: X \times X \rightarrow X$ and $g: X \rightarrow X$ are called $W$-compatible if $g(F(x, y))=F(g x, g y)$ and $g(F(y, x))=$ $F(g y, g x)$ whenever $g x=F(x, y)$ and $g y=F(y, x)$ for some $(x, y) \in X \times X$.

Now, we give our main results.

\section{Main Results}

Theorem 18. Let $(X, G, *)$ be a G-fuzzy metric space with $a *$ $b=\min \{a, b\}$ for all $a, b \in[0,1]$ and $S: X \times X \rightarrow X$ and let $f: X \rightarrow X$ be mappings satisfying

$$
\begin{aligned}
& G(S(x, y), S(u, v), S(u, v), k t) \\
& \quad \geq \min \{G(f x, f u, f u, t), G(f y, f v, f v, t)\}
\end{aligned}
$$

for all $x, y, u, v \in X$, where $0 \leq k<1$,

$S(X \times X) \subseteq f(X)$ and $f(X)$ is a complete subspace of $X$, the pair $(f, S)$ is $W$-compatible.

Then $S$ and $f$ have a unique common coupled fixed point of the form $(\alpha, \alpha)$ in $X \times X$.

Proof. Let $x_{0}, y_{0} \in X$ and denote $z_{n}=S\left(x_{n}, y_{n}\right)=$ $f x_{n+1}, p_{n}=S\left(y_{n}, x_{n}\right)=f y_{n+1}, n=0,1,2, \ldots$. Let $d_{n}(t)=$ 
$G\left(z_{n}, z_{n+1}, z_{n+1}, t\right), e_{n}(t)=G\left(p_{n}, p_{n+1}, p_{n+1}, t\right)$. From (2), we have

$$
\begin{aligned}
d_{n+1}(k t) & =G\left(z_{n+1}, z_{n+2}, z_{n+2}, k t\right) \\
& =G\left(S\left(x_{n+1}, y_{n+1}\right), S\left(x_{n+2}, y_{n+2}\right), S\left(x_{n+2}, y_{n+2}\right), k t\right) \\
& \geq \min \left\{G\left(z_{n}, z_{n+1}, z_{n+1}, t\right), G\left(p_{n}, p_{n+1}, p_{n+1}, t\right)\right\} \\
& \geq \min \left\{d_{n}(t), e_{n}(t)\right\} .
\end{aligned}
$$

Also,

$$
\begin{aligned}
e_{n+1}(k t) & =G\left(p_{n+1}, p_{n+2}, p_{n+2}, k t\right) \\
& =G\left(S\left(y_{n+1}, x_{n+1}\right), S\left(y_{n+2}, x_{n+2}\right), S\left(y_{n+2}, x_{n+2}\right), k t\right) \\
& \geq \min \left\{G\left(p_{n}, p_{n+1}, p_{n+1}, t\right), G\left(z_{n}, z_{n+1}, z_{n+1}, t\right)\right\} \\
& \geq \min \left\{e_{n}(t), d_{n}(t)\right\} .
\end{aligned}
$$

Thus, $\min \left\{d_{n+1}(k t), e_{n+1}(k t)\right\} \geq \min \left\{d_{n}(t), e_{n}(t)\right\}$. Hence,

$$
\begin{aligned}
\min & \left\{d_{n}(t), e_{n}(t)\right\} \\
& \geq \min \left\{d_{n-1}\left(\frac{t}{k}\right), e_{n-1}\left(\frac{t}{k}\right)\right\} \\
& \geq \min \left\{d_{n-2}\left(\frac{t}{k^{2}}\right), e_{n-2}\left(\frac{t}{k^{2}}\right)\right\} \\
& \vdots \\
& \geq \min \left\{d_{0}\left(\frac{t}{k^{n}}\right), e_{0}\left(\frac{t}{k^{n}}\right)\right\} \\
& =\min \left\{G\left(z_{0}, z_{1}, z_{1}, \frac{t}{k^{n}}\right), G\left(p_{0}, p_{1}, p_{1}, \frac{t}{k^{n}}\right)\right\} .
\end{aligned}
$$

For any positive integer $n$ and fixed positive integer $p$, we have

$$
\begin{aligned}
& G\left(z_{n}, z_{n+p}, z_{n+p}, t\right) \\
& \geq G\left(z_{n+p-1}, z_{n+p}, z_{n+p}, \frac{t}{p}\right) * G\left(z_{n+p-2}, z_{n+p-1}, z_{n+p-1}, \frac{t}{p}\right) \\
& \quad * \cdots * G\left(z_{n}, z_{n+1}, z_{n+1}, \frac{t}{p}\right) \\
& \geq \min \left\{G\left(z_{0}, z_{1}, z_{1}, \frac{t}{p k^{n+p-1}}\right), G\left(p_{0}, p_{1}, p_{1}, \frac{t}{p k^{n+p-1}}\right)\right\} \\
& \quad * \min \left\{G\left(z_{0}, z_{1}, z_{1}, \frac{t}{p k^{n+p-2}}\right), G\left(p_{0}, p_{1}, p_{1}, \frac{t}{p k^{n+p-2}}\right)\right\} \\
& \quad * \cdots * \min \left\{G\left(z_{0}, z_{1}, z_{1}, \frac{t}{p k^{n}}\right), G\left(p_{0}, p_{1}, p_{1}, \frac{t}{p k^{n}}\right)\right\} .
\end{aligned}
$$

Letting $n \rightarrow \infty$ and using $(\mathrm{P})$, we get

$$
\lim _{n \rightarrow \infty} G\left(z_{n}, z_{n+p}, z_{n+p}, t\right) \geq 1 * 1 * \cdots * 1=1 .
$$

Hence, $\lim _{n \rightarrow \infty} G\left(z_{n}, z_{n+p}, z_{n+p}, t\right)=1$. Thus, $\left\{z_{n}\right\}$ is $G^{-}$ Cauchy in $X$. Similarly, we can show that $\left\{p_{n}\right\}$ is G-Cauchy in $X$. Since $f(X)$ is $G$-complete, $\left\{z_{n}\right\}$ and $\left\{p_{n}\right\}$ converge to some $\alpha$ and $\beta$ in $f(X)$, respectively. Hence, there exist $x$ and $y$ in $X$ such that $\alpha=f x, \beta=f y$ :

$$
\begin{aligned}
G\left(z_{n}, S(x, y), S(x, y), k t\right) \\
\quad=G\left(S\left(x_{n}, y_{n}\right), S(x, y), S(x, y), k t\right) \\
\quad \geq \min \left\{G\left(z_{n-1}, f x, f x, t\right), G\left(p_{n-1}, f y, f y, t\right)\right\} .
\end{aligned}
$$

Letting $n \rightarrow \infty$, we get

$$
\mathrm{G}(f x, S(x, y), S(x, y), k t) \geq \min \{1,1\}=1 .
$$

Hence, $S(x, y)=f x$. Similarly, it can be shown that $S(y, x)=$ $f y$. Since $(f, S)$ is $W$-compatible, we have

$$
\begin{gathered}
f \alpha=f f x=f(S(x, y))=S(f x, f y)=S(\alpha, \beta) \\
f \beta=f f y=f(S(y, x))=S(f y, f x)=S(\beta, \alpha) . \\
G\left(z_{n}, f \alpha, f \alpha, k t\right) \\
=G\left(S\left(x_{n}, y_{n}\right), S(\alpha, \beta), S(\alpha, \beta), k t\right) \\
\geq \min \left\{G\left(z_{n-1}, f \alpha, f \alpha, t\right), G\left(p_{n-1}, f \beta, f \beta, t\right)\right\} .
\end{gathered}
$$

Letting $n \rightarrow \infty$, we get

$G(\alpha, f \alpha, f \alpha, k t) \geq \min \{G(\alpha, f \alpha, f \alpha, t), G(\beta, f \beta, f \beta, t)\}$.

Similarly, we can show that

$$
G(\beta, f \beta, f \beta, k t) \geq \min \{G(\alpha, f \alpha, f \alpha, t), G(\beta, f \beta, f \beta, t)\} .
$$

Thus,

$$
\begin{aligned}
& \min \{G(\alpha, f \alpha, f \alpha, k t), G(\beta, f \beta, f \beta, k t)\} \\
& \geq \min \{G(\alpha, f \alpha, f \alpha, t), G(\beta, f \beta, f \beta, t)\} .
\end{aligned}
$$

From Lemma 14, we have $f \alpha=\alpha$ and $f \beta=\beta$. Thus, $\alpha=f \alpha=S(\alpha, \beta)$ and $\beta=f \beta=S(\beta, \alpha)$. Hence, $(\alpha, \beta)$ is a common coupled fixed point of $S$ and $f$.

Suppose $\left(\alpha^{1}, \beta^{1}\right)$ is another common coupled fixed point of $S$ and $f$ :

$$
\begin{aligned}
G\left(\alpha, \alpha^{1}, \alpha^{1}, k t\right) & =G\left(S(\alpha, \beta), S\left(\alpha^{1}, \beta^{1}\right), S\left(\alpha^{1}, \beta^{1}\right), k t\right) \\
& \geq \min \left\{G\left(\alpha, \alpha^{1}, \alpha^{1}, t\right), G\left(\beta, \beta^{1}, \beta^{1}, t\right)\right\}
\end{aligned}
$$

Similarly,

$$
\begin{aligned}
G\left(\beta, \beta^{1}, \beta^{1}, k t\right) & =G\left(S(\beta, \alpha), S\left(\beta^{1}, \alpha^{1}\right), S\left(\beta^{1}, \alpha^{1}\right), k t\right) \\
& \geq \min \left\{G\left(\alpha, \alpha^{1}, \alpha^{1}, t\right), G\left(\beta, \beta^{1}, \beta^{1}, t\right)\right\} .
\end{aligned}
$$


Thus,

$$
\begin{aligned}
\min & \left\{G\left(\alpha, \alpha^{1}, \alpha^{1}, k t\right), G\left(\beta, \beta^{1}, \beta^{1}, k t\right)\right\} \\
& \geq \min \left\{G\left(\alpha, \alpha^{1}, \alpha^{1}, t\right), G\left(\beta, \beta^{1}, \beta^{1}, t\right)\right\} .
\end{aligned}
$$

From Lemma $14, \alpha^{1}=\alpha$ and $\beta^{1}=\beta$. Thus, $(\alpha, \beta)$ is the unique common coupled fixed point of $S$ and $f$. Now, we will show that $\alpha=\beta$ :

$$
\begin{aligned}
G(\alpha, \alpha, \beta, k t) & =G(S(\alpha, \beta), S(\alpha, \beta), S(\beta, \alpha), k t) \\
& \geq \min \{G(\alpha, \alpha, \beta, t), G(\beta, \beta, \alpha, t)\}, \\
G(\alpha, \beta, \beta, k t) & =G(S(\alpha, \beta), S(\beta, \alpha), S(\beta, \alpha), k t) \\
& \geq \min \{G(\alpha, \beta, \beta, t), G(\beta, \alpha, \alpha, t)\} .
\end{aligned}
$$

Thus,

$$
\begin{aligned}
\min & \{G(\alpha, \alpha, \beta, k t), G(\alpha, \beta, \beta, k t)\} \\
& \geq \min \{G(\alpha, \alpha, \beta, t), G(\alpha, \beta, \beta, t)\} .
\end{aligned}
$$

From Lemma 14 , we have $\alpha=\beta$. Thus, $\alpha$ is a common fixed point of $S$ and $f$, that is, $\alpha=f \alpha=S(\alpha, \alpha)$. Suppose $\alpha^{1}$ is another common fixed point of $S$ and $f$ :

$$
\begin{aligned}
G\left(\alpha^{1}, \alpha, \alpha, t\right) & =G\left(S\left(\alpha^{1}, \alpha^{1}\right), S(\alpha, \alpha), S(\alpha, \alpha), t\right) \\
& \geq \min \left\{G\left(\alpha^{1}, \alpha, \alpha, \frac{t}{k}\right), G\left(\alpha^{1}, \alpha, \alpha, \frac{t}{k}\right)\right\} \\
& \geq G\left(\alpha^{1}, \alpha, \alpha, \frac{t}{k^{2}}\right) \\
& \vdots \\
& \geq G\left(\alpha^{1}, \alpha, \alpha, \frac{t}{k^{n}}\right) \longrightarrow 1 \quad \text { as } n \longrightarrow \infty
\end{aligned}
$$

Hence, $\alpha^{1}=\alpha$. Thus, $S$ and $f$ have a unique common coupled fixed point of the form $(\alpha, \alpha)$.

Finally, we prove a common coupled fixed-point theorem for three mappings in symmetric $G$-fuzzy metric spaces.

Theorem 19. Let $(X, G, *)$ be a symmetric $G$-complete fuzzy metric space with $a * b=\min \{a, b\}$ for all $a, b \in[0,1]$ and let $S, T, R: X \times X \rightarrow X$ be mappings satisfying

$$
\begin{gathered}
G(S(x, y), T(u, v), R(p, q), k t) \\
\geq \min \{G(x, u, p, t), G(y, v, q, t), G(x, x, S(x, y), t), \\
G(u, u, T(u, v), t), G(p, p, R(p, q), t)\}
\end{gathered}
$$

for all $x, y, u, v, p, q \in X$, where $0 \leq k<1$. Then, there exists $(x, y) \in X \times X$ such that

$$
\begin{aligned}
& x=S(x, y)=T(x, y)=R(x, y), \\
& y=S(y, x)=T(y, x)=R(y, x) .
\end{aligned}
$$

Or

$S, T$, and $R$ have a unique common coupled fixed point

of the form $(x, x)$ in $X \times X$.

Proof. Let $x_{0}, y_{0} \in X$. Define the sequences $\left\{x_{n}\right\}$ and $\left\{y_{n}\right\}$ in $X$ as follows: $x_{3 n+1}=S\left(x_{3 n}, y_{3 n}\right), y_{3 n+1}=S\left(y_{3 n}, x_{3 n}\right)$; $x_{3 n+2}=T\left(x_{3 n+1}, y_{3 n+1}\right), y_{3 n+2}=T\left(y_{3 n+1}, x_{3 n+1}\right) ; x_{3 n+3}=$ $R\left(x_{3 n+2}, y_{3 n+2}\right), y_{3 n+3}=R\left(y_{3 n+2}, x_{3 n+2}\right), n=0,1,2, \ldots$ Suppose $x_{3 n+1}=x_{3 n}$ for some $n$. Then, $S(x, y)=x$, where $x=x_{3 n}, y=y_{3 n}$. Suppose $T(x, y) \neq R(x, y)$. Then,

$$
\begin{aligned}
G(x, & T(x, y), R(x, y), k t) \\
& =G(S(x, y), T(x, y), R(x, y), k t) \\
& \geq \min \{1,1,1, G(x, x, T(x, y), t), G(x, x, R(x, y), t)\} \\
& \geq G(x, T(x, y), R(x, y), t) .
\end{aligned}
$$

It is a contradiction. Hence, $T(x, y)=R(x, y)$. From (25) and since $X$ is symmetric,

$$
\begin{aligned}
G(x, T(x, y), T(x, y), k t) & \geq G(x, x, T(x, y), t) \\
& =G(x, T(x, y), T(x, y), t) .
\end{aligned}
$$

From Lemma 14, we have $T(x, y)=x$. Thus, $S(x, y)=$ $T(x, y)=R(x, y)=x$. Similarly, if $x_{3 n+1}=x_{3 n+2}$ or $x_{3 n+2}=x_{3 n+3}$, then also we can show that $S(x, y)=T(x, y)=$ $R(x, y)=x$ for some $x, y$ in $X$. Similarly, it can be shown that if $y_{3 n}=y_{3 n+1}$ or $y_{3 n+1}=y_{3 n+2}$ or $y_{3 n+2}=y_{3 n+3}$ then there exists $(x, y) \in X \times X$ such that

$$
S(y, x)=T(y, x)=R(y, x)=y .
$$

Now, assume that $x_{n} \neq x_{n+1}$ and $y_{n} \neq y_{n+1}$ for all $n$. Write $d_{n}(t)=G\left(x_{n}, x_{n+1}, x_{n+2}, t\right)$ and $e_{n}(t)=G\left(y_{n}, y_{n+1}, y_{n+2}, t\right)$ :

$$
\begin{aligned}
& d_{3 n}(k t) \\
& =G\left(x_{3 n}, x_{3 n+1}, x_{3 n+2}, k t\right) \\
& =G\left(S\left(x_{3 n}, y_{3 n}\right), T\left(x_{3 n+1}, y_{3 n+1}\right), R\left(x_{3 n-1}, y_{3 n-1}\right), k t\right) \\
& \geq \min \left\{d_{3 n-1}(t), e_{3 n-1}(t), G\left(x_{3 n}, x_{3 n}, x_{3 n+1}, t\right),\right. \\
& \left.\quad G\left(x_{3 n+1}, x_{3 n+1}, x_{3 n+2}, t\right), G\left(x_{3 n-1}, x_{3 n-1}, x_{3 n}, t\right)\right\} \\
& \geq \min \left\{d_{3 n-1}(t), e_{3 n-1}(t), d_{3 n}(t), d_{3 n}(t), d_{3 n-1}(t)\right\} .
\end{aligned}
$$

Thus, $d_{3 n}(k t) \geq \min \left\{d_{3 n-1}(t), e_{3 n-1}(t)\right\}$. Similarly, we have $e_{3 n}(k t) \geq \min d_{3 n-1}(t), e_{3 n-1}(t)$.

Thus,

$$
\min \left\{d_{3 n}(k t), e_{3 n}(k t)\right\} \geq \min \left\{d_{3 n-1}(t), e_{3 n-1}(t)\right\} .
$$

Similarly, we can show that

$$
\begin{gathered}
\min \left\{d_{3 n+1}(k t), e_{3 n+1}(k t)\right\} \geq \min \left\{d_{3 n}(t), e_{3 n}(t)\right\}, \\
\min \left\{d_{3 n+2}(k t), e_{3 n+2}(k t)\right\} \geq \min \left\{d_{3 n+1}(t), e_{3 n+1}(t)\right\} .
\end{gathered}
$$


Thus,

$$
\min \left\{d_{n+1}(k t), e_{n+1}(k t)\right\} \geq \min \left\{d_{n}(t), e_{n}(t)\right\} .
$$

Hence

$$
\begin{aligned}
\min & \left\{d_{n}(t), e_{n}(t)\right\} \\
& \geq \min \left\{d_{n}\left(\frac{t}{k}\right), e_{n}\left(\frac{t}{k}\right)\right\} \\
& \geq \min \left\{d_{n}\left(\frac{t}{k^{2}}\right), e_{n}\left(\frac{t}{k^{2}}\right)\right\} \\
& \vdots \\
& \geq \min \left\{d_{0}\left(\frac{t}{k^{n}}\right), e_{0}\left(\frac{t}{k^{n}}\right)\right\} \\
& =\min \left\{G\left(x_{0}, x_{1}, x_{2}, \frac{t}{k^{n}}\right), G\left(y_{0}, y_{1}, y_{2}, \frac{t}{k^{n}}\right)\right\} .
\end{aligned}
$$

Thus,

$$
\begin{aligned}
& G\left(x_{n}, x_{n+1}, x_{n+2}, t\right) \\
& \quad \geq \min \left\{G\left(x_{0}, x_{1}, x_{2}, \frac{t}{k^{n}}\right), G\left(y_{0}, y_{1}, y_{2}, \frac{t}{k^{n}}\right)\right\} .
\end{aligned}
$$

From $\left(G_{3}\right)$, we have

$$
\begin{aligned}
G\left(x_{n}, x_{n}, x_{n+1}, t\right) \\
\quad \geq G\left(x_{n}, x_{n+1}, x_{n+2}, t\right) \\
\quad \geq \min \left\{G\left(x_{0}, x_{1}, x_{2}, \frac{t}{k^{n}}\right), G\left(y_{0}, y_{1}, y_{2}, \frac{t}{k^{n}}\right)\right\} .
\end{aligned}
$$

As in Theorem 18, we can show that $\left\{x_{n}\right\}$ and $\left\{y_{n}\right\}$ are $G$ Cauchy sequences in $X$. Since $X$ is $G$-complete, there exist $x, y \in X$ such that $x_{n} \rightarrow x$ and $y_{n} \rightarrow y$ :

$$
\begin{gathered}
G\left(S(x, y), x_{3 n+2}, x_{3 n+3}, k t\right) \\
=G\left(S(x, y), T\left(x_{3 n+1}, y_{3 n+1}\right), R\left(x_{3 n+2}, y_{3 n+2}\right), k t\right) \\
\geq \min \left\{G\left(x, x_{3 n+1}, x_{3 n+2}, t\right), G\left(y, y_{3 n+1}, y_{3 n+2}, t\right),\right. \\
G(x, x, S(x, y), t), G\left(x_{3 n+1}, x_{3 n+1}, x_{3 n+2}, t\right), \\
\left.G\left(x_{3 n+2}, x_{3 n+2}, x_{3 n+3}, t\right)\right\} .
\end{gathered}
$$

Letting $n \rightarrow \infty$,

$$
\begin{aligned}
G(S(x, y), x, x, k t) & \geq \min \{1,1, G(x, x, S(x, y), t), 1,1\} \\
& =G(x, x, S(x, y), t) .
\end{aligned}
$$

From this, we have $S(x, y)=x$. As in the first part of proof, we can show that $S(x, y)=T(x, y)=R(x, y)=x$. Similarly, it can be shown that $S(y, x)=T(y, x)=R(y, x)=y$. Thus, $(x, y)$ is a common coupled fixed point of $S, T$, and
$R$. Suppose $\left(x^{1}, y^{1}\right)$ is another common coupled fixed point of $S, T$, and $R$. Consider

$$
\begin{aligned}
G\left(x, x, x^{1}, k t\right) & =G\left(S(x, y), T(x, y), R\left(x^{1}, y^{1}\right), k t\right) \\
& \geq \min \left\{G\left(x, x, x^{1}, t\right), G\left(y, y, y^{1}, t\right), 1,1,1\right\} \\
& =\min \left\{G\left(x, x, x^{1}, t\right), G\left(y, y, y^{1}, t\right)\right\} .
\end{aligned}
$$

Also,

$$
\begin{aligned}
G\left(y, y, y^{1}, k t\right) & =G\left(S(y, x), T(y, x), R\left(y^{1}, x^{1}\right), k t\right) \\
& \geq \min \left\{G\left(x, x, x^{1}, t\right), G\left(y, y, y^{1}, t\right), 1,1,1\right\} \\
& =\min \left\{G\left(x, x, x^{1}, t\right), G\left(y, y, y^{1}, t\right)\right\} .
\end{aligned}
$$

Thus,

$$
\begin{aligned}
\min & \left\{G\left(x, x, x^{1}, k t\right), G\left(y, y, y^{1}, k t\right)\right\} \\
& \geq \min \left\{G\left(x, x, x^{1}, t\right), G\left(y, y, y^{1}, t\right)\right\} .
\end{aligned}
$$

From Lemma 14, we have $x^{1}=x$ and $y^{1}=y$. Thus, $(x, y)$ is the unique common coupled fixed point of $S, T$, and $R$. Now, we will show that $x=y$. Consider

$$
\begin{aligned}
G(x, x, y, k t) & =G(S(x, y), T(x, y), R(y, x), k t) \\
& \geq \min \{G(x, x, y, t) G(y, y, x, t), 1,1,1\} \\
& =G(x, x, y, t) .
\end{aligned}
$$

Hence, $x=y$. Thus, $S, T$, and $R$ have a unique common coupled fixed point of the form $(x, x)$.

\section{Acknowledgment}

The authors are thankful to the referee for his valuable suggestions.

\section{References}

[1] Z. Mustafa and B. Sims, "Some remarks concerninig D-metric spaces," in Proceedings of the Internatinal Conferences on Fixed Point Theory and Applications, pp. 189-198, Valencia, Spain, July 2003.

[2] Z. Mustafa and B. Sims, "A new approach to generalized metric spaces," Journal of Nonlinear and Convex Analysis, vol. 7, no. 2, pp. 289-297, 2006.

[3] Z. Mustafa and B. Sims, "Fixed point theorems for contractive mappings in complete G-metric spaces," Fixed Point Theory and Applications, vol. 2009, Article ID 917175, 10 pages, 2009.

[4] S. V. R. Naidu, K. P. R. Rao, and N. Srinivasa Rao, "On convergent sequences and fixed point theorems in $D$-metric spaces," International Journal of Mathematics and Mathematical Sciences, no. 12, pp. 1969-1988, 2005.

[5] B. C. Dhage, "Generalized metric spaces and mapping with fixed points," Bulletin of the Calcutta Mathematical Society, vol. 84, pp. 329-336, 1992. 
[6] B. C. Dhage, "On generalized metric spaces and topological structure II," Pure and Applied Mathematika Sciences, vol. 40, no. 1-2, pp. 37-41, 1994.

[7] B. C. Dhage, "A common fixed point principle in D-metric spaces," Bulletin of the Calcutta Mathematical Society, vol. 91, no. 6, pp. 475-480, 1999.

[8] B. C. Dhage, "Generalized metric spaces and topological structure. I," Annalele Stiintifice ale Universitatii Al.I.Cuza, vol. 46, no. 1, pp. 3-24, 2000.

[9] M. Abbas and B. E. Rhoades, "Common fixed point results for noncommuting mappings without continuity in generalized metric spaces," Applied Mathematics and Computation, vol. 215, no. 1, pp. 262-269, 2009.

[10] R. Chugh, T. Kadian, A. Rani, and B. E. Rhoades, "Property P in G-metric spaces," Fixed Point Theory and Applications, vol. 2010, Article ID 401684, 12 pages, 2010.

[11] Z. Mustafa, F. Awawdeh, and W. Shatanawi, "Fixed point theorem for expansive mappings in G-metric spaces," International Journal of Contemporary Mathematical Sciences, vol. 5, no. 4952, pp. 2463-2472, 2010.

[12] Z. Mustafa and H. Obiedat, "A fixed point theorem of Reich in G-metric spaces," Cubo A Mathematical Journal, vol. 12, no. 1, pp. 83-93, 2010.

[13] Z. Mustafa, H. Obiedat, and F. Awawdeh, "Some fixed point theorem for mapping on complete G-metric spaces," Fixed Point Theory and Applications, vol. 2008, Article ID 189870, 12 pages, 2008.

[14] Z. Mustafa, W. Shatanawi, and M. Bataineh, "Existence of fixed point results in G-metric spaces," International Journal of Mathematics and Mathematical Sciences, vol. 2009, Article ID 283028, 10 pages, 2009.

[15] W. Shatanawi, "Fixed point theory for contractive mappings satisfying $\Phi$-maps in G-metric spaces," Fixed Point Theory and Applications, vol. 2010, Article ID 181650, 9 pages, 2010.

[16] G. Sun and K. Yang, "Generalized fuzzy metric spaces with properties," Research journal of Applied Sciences, Engineering and Technology, vol. 2, no. 7, pp. 673-678, 2010.

[17] T. G. Bhaskar and V. Lakshmikantham, "Fixed point theorems in partially ordered metric spaces and applications," Nonlinear Analysis, vol. 65, no. 7, pp. 1379-1393, 2006.

[18] M. Abbas, M. Ali Khan, and S. Radenović, "Common coupled fixed point theorems in cone metric spaces for W-compatible mappings," Applied Mathematics and Computation, vol. 217, no. 1, pp. 195-202, 2010. 

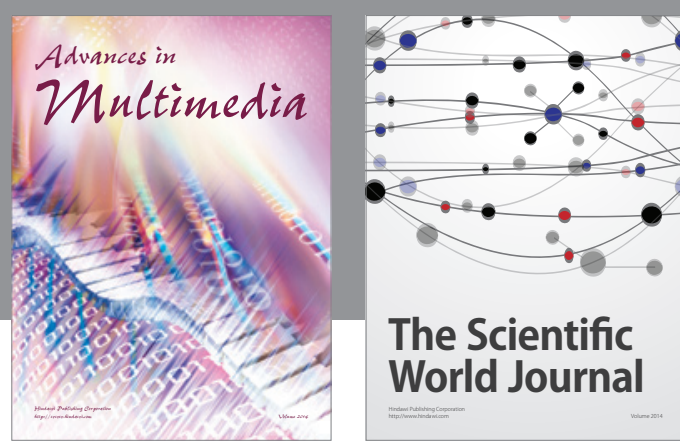

The Scientific World Journal
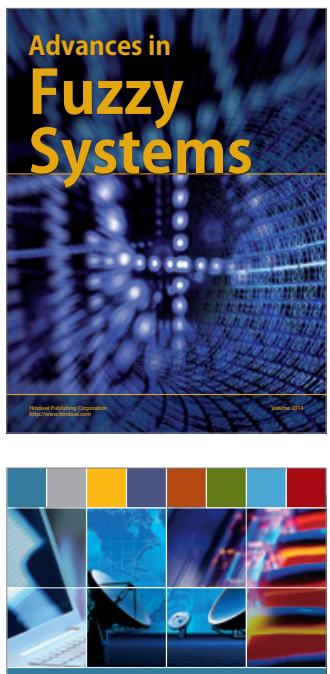

Computer Networks and Communications
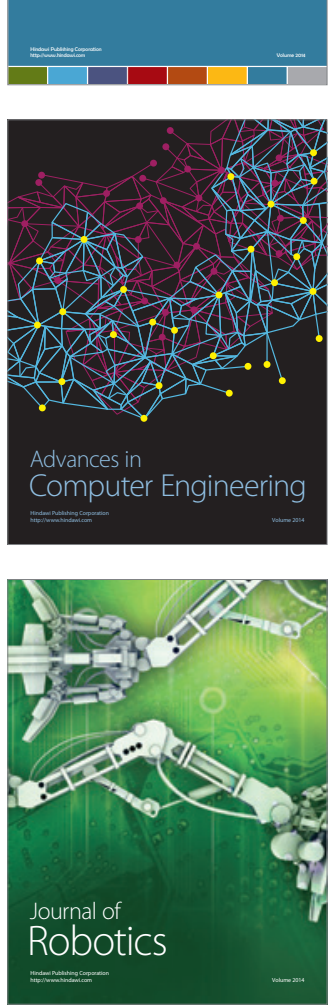
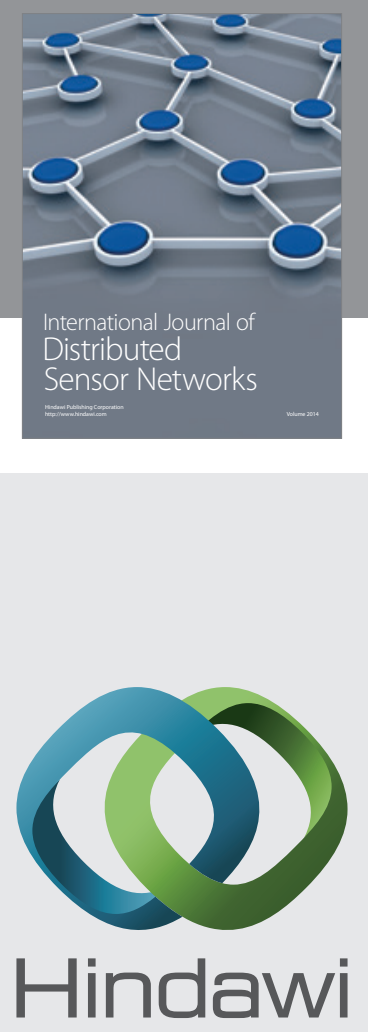

Submit your manuscripts at

http://www.hindawi.com
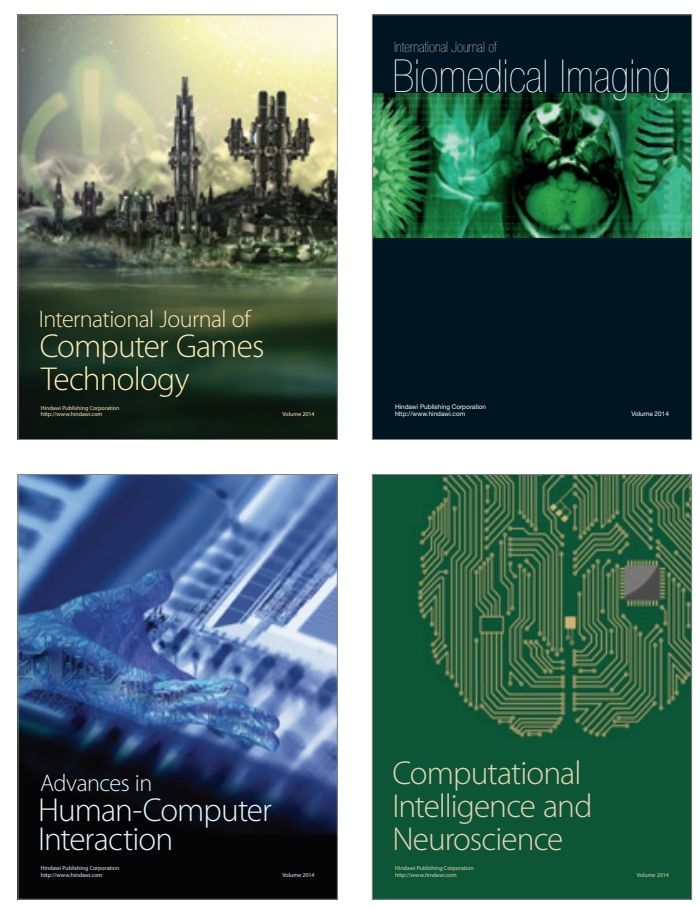
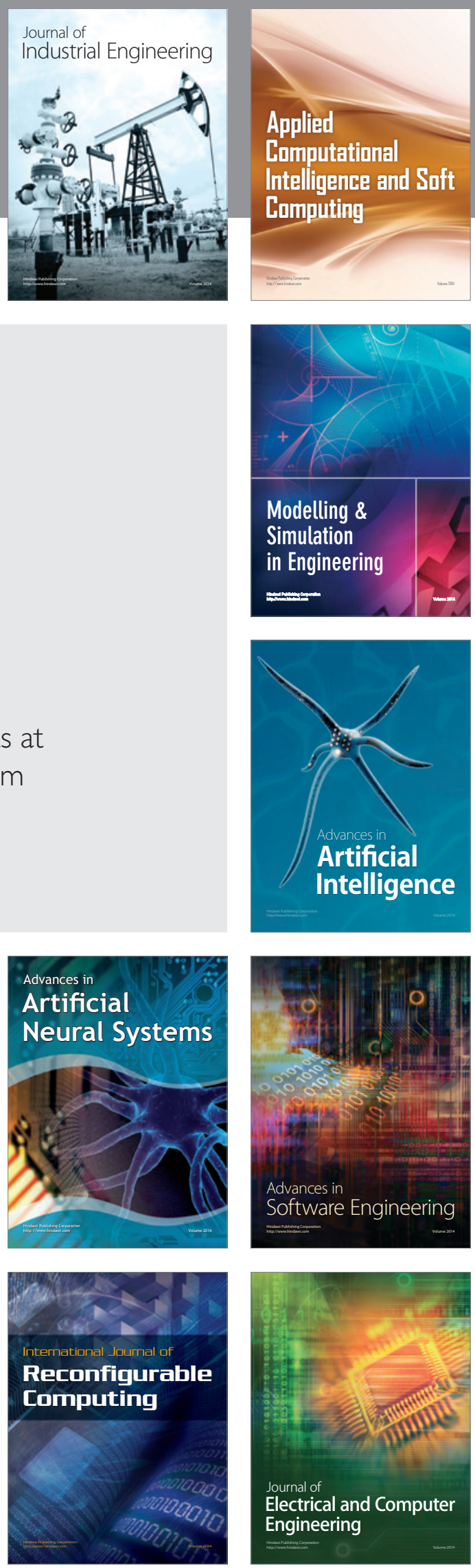